\title{
Refining the UKST H $\alpha$ Survey's PN Database Using MSX
}

\author{
Martin Cohen \\ Radio Astronomy Laboratory, 601 Campbell Hall, University of \\ California, Berkeley, CA 94720, USA \\ Quentin A. Parker ${ }^{1,2}$ \\ ${ }^{1}$ Wide Field Astronomy Unit, Institute for Astronomy, University of \\ Edinburgh, Edinburgh EH9 3HJ, Scotland, UK; ${ }^{2}$ Dept.Physics, \\ University of Macquarie, NSW 21090, Australia
}

\begin{abstract}
We are combining images in the mid-infrared (from MSX, the US Midcourse Space Experiment) with those from the high resolution $\mathrm{UKST} / \mathrm{AAO} \mathrm{H} \alpha$ survey of the Galactic Plane to investigate the relationship between the mid-infrared (MIR) and optical emission line properties of candidate Planetary Nebulae (PN) discovered by the $\mathrm{H} \alpha$ survey. Preliminary results indicate that only $10-20 \%$ of likely bona fide $\mathrm{PN}$ have a counterpart detected in the MIR. By sharp contrast one can readily recognize pre-main- sequence objects and HII regions emitting $\mathrm{H} \alpha$ and largely embedded in dust by the simultaneous presence of 8.3 and 21.3$\mu \mathrm{m}$ emission suggestive of dust spanning a variety of temperatures. The sharpest criteria for recognizing true PN appear to be optical morphology and spectra (particularly the presence of [OIII]), and, where detected, MIR characteristics.
\end{abstract}

\section{Introduction}

The US Midcourse Space Experiment (MSX: Mill et al. 1994) was a mission of the Ballistic Missile Defense Organization and later of the USAF. It accomplished a great deal of mid-infrared (MIR) astronomy using the Spatial InfraRed Imaging Telescope (SPIRIT-III) with radiometers covering the range 4.3-21.3 $\mu \mathrm{m}$, principally an imaging survey of the entire Galactic Plane for $|b| \leq 5^{\circ}$. This major panoramic survey, with a spatial resolution of $20^{\prime \prime}$ in all four MIR wavelengths $(8.3,12.1,14.6,21.3 \mu \mathrm{m})$ and now publicly available through IPAC (Pasadena), is described by Price et al. (2001).

The rationale for the choice of SPIRIT-III bands can best be understood by comparing the MSX relative response curves with the MIR emission from the general interstellar medium (ISM), as typified by the emission spectrum of a reflection nebula, which results when a random gas and dust cloud in the ISM is illuminated by the UV radiation field. The ubiquitous polycyclic aromatic hydrocarbons (PAHs: containing tens of C-atoms) in that cloud fluoresce through several MIR emission bands, the strongest of which $(6.2,7.7,8.7$, with 
the underlying 6-9 $\mu \mathrm{m}$ plateau of small carbon particles, containing hundreds of C-atoms) lie within MSX's most sensitive band (" $\mathrm{A}$ ": at an isophotal wavelength of $8.3 \mu \mathrm{m}$ ), as do silicate dust bands, while the $11.3-\mu \mathrm{m}$ PAH band and the plateau from 10-14 $\mu \mathrm{m}$, fall within "Band-C" $\left(\lambda_{\text {iso }} 12.1 \mu \mathrm{m}\right)$. "Band-D" $\left(\lambda_{\text {iso }}\right.$ $14.6 \mu \mathrm{m}$ ) was originally chosen to sample non-silicate dust grains. It has subsequently been found also to include PAH features, near $16.4 \mu \mathrm{m}$, but these are neither frequently encountered nor strong, so the original purpose still remains. The longest and least sensitive "Band-E" ( $\left.\lambda_{\text {iso }} 21.3 \mu \mathrm{m}\right)$ probes the $\sim 19-\mu \mathrm{m}$ resonance of amorphous silicate grains in the ISM.

Because SPIRIT-III was equally sensitive to both diffuse and point source MIR emission, and because photodissociation regions (PDRs) are bright in PAH emission, MSX was ideally designed to study both large scale PDRs, which are found as interfaces in the ISM, and smaller scale versions such as those that surround HII regions and PN.

\section{PN in the MIR}

PN can be bright MIR objects because of PAH emission, atomic fine structure lines, $\mathrm{H}_{2}$ rotational lines, and/or thermal dust emission within the nebulae (powered by resonantly trapped Ly $\alpha$ photons) and in circumnuclear disks (powered by dilute starlight). The history of MIR observations of PN from the ground can be traced through the papers of Cohen \& Barlow (1974;1980), Aitken et al. (1979), and recently, for a sample of bulge PN, Casassus et al. (2001). The recognition of the importance of the major $\mathrm{PAH}$ bands required airborne spectroscopy (Cohen et al. 1986,1989), while IRAS offered a substantial number of broadband measurements of PN as well as low-resolution spectra of many (Volk \& Cohen 1990). Cox et al. (1998) observed that the Helix's MIR emission is due to pure rotational lines of $\mathrm{H}_{2}$.

\section{MSX \& the UKST H $\alpha$ Survey of the Southern Galactic Plane}

To assess the potential value of seeking MSX counterpart images to all the new UKST PN candidates discovered from the AAO/UKST $\mathrm{H} \alpha$ survey of the Southern Galactic Plane (see Parker et al. these proceedings) we took an earlier (Nov. 2000) version of the database that comprised 497 candidate objects, and defined a selection of 142 objects, in several distinct subsets, for which we sought 8.3 and $21.3-\mu \mathrm{m}$ MSX images. The sub-samples were defined as follows: a bulge field, HA17685; an inner Galaxy, non-bulge field, HA17901, at $l=300^{\circ} ; 4$ of the largest (angular size) PN found by the $\mathrm{H} \alpha$ Survey; 10 new bona fide PN (based on optical spectra and morphology); $3 \mathrm{PN}$ with Wolf-Rayet nuclei; a set of candidate $\mathrm{PN}$ with bipolar or odd morphologies; and a large random sample of new candidates with $|b| \leq 0.75^{\circ}$. In this way we hoped to sample the range of environments and candidate types encountered.

We present several examples of these candidates, comparing their MIR and optical structures and dimensions, and describing optical spectroscopy of the nebulae. 


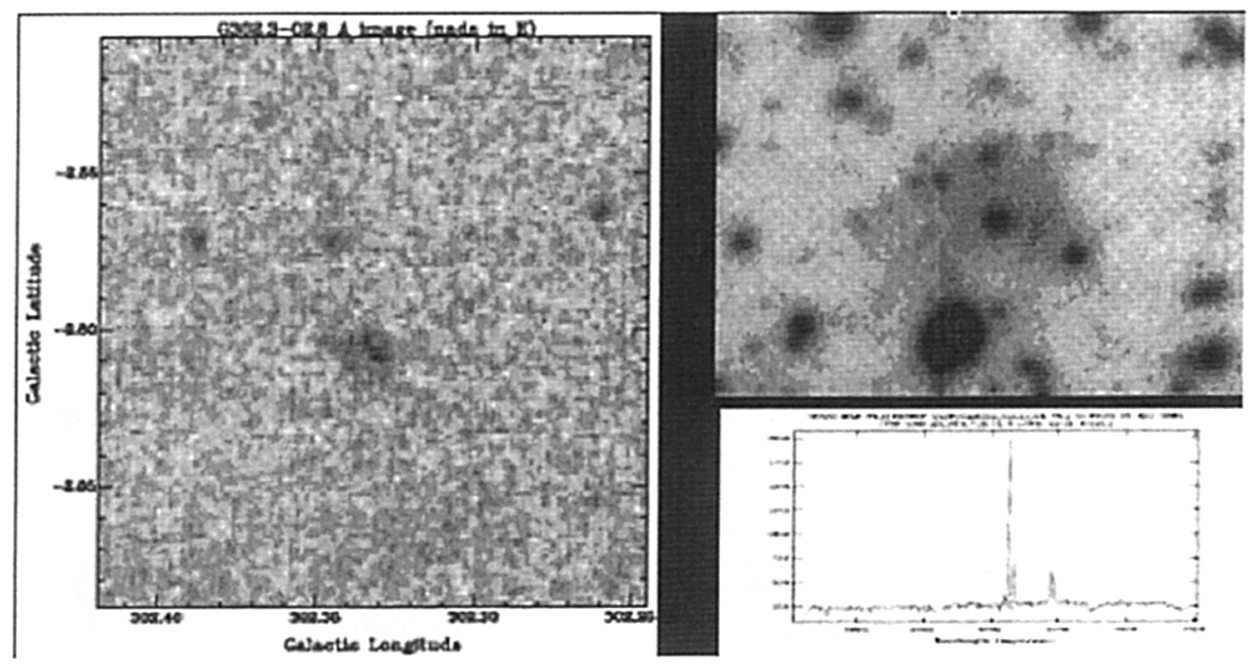

Figure 1. MSX 8.3- $\mu \mathrm{m}$ (left) and $\mathrm{H} \alpha$ (top right) images of PNG 302.3-02.6, and the red spectral lines (bottom right).

\subsection{H $\alpha$ survey PN candidates with MSX counterparts}

PNG 302.3-02.6 (Figure 1) has an $\mathrm{H} \alpha$ diameter of $23^{\prime \prime}$, a red spectrum showing $\mathrm{H} \alpha$, [NII] and [SII], and is a diffuse 8.3- $\mu \mathrm{m}$ emission source.

PNG 303.1-00.9 (Figure 2) was selected because of its clear bipolar morphology in $\mathrm{H} \alpha$, where one sees a $26^{\prime \prime}$ nebula, with two lobes surrounding a central elliptical blob or star. MSX reveals a very compact, bright, extended object at 8.3 and $21.3 \mu \mathrm{m}$, indicative of the brightness of the central star though very faint 8.3- $\mu \mathrm{m}$ emission attends the lobes too. This PN epitomizes the known bias toward MIR brightness of bipolar nebulae. This morphology arises from an extensive circumstellar dusty disk surrounding the central star, with grains heated by dilute starlight and thus spanning a wide range of temperature. It is also interesting to see the unique way in which MSX imagery reveals the environs of such PN: note the large 8.3- $\mu$ m loop in Figure 2 which suggests that PNG 303.100.9 did not form in isolation but perhaps along the meandering surface of an extended bubble, emitting in the MIR because it is a PDR, cooling through the PAH emission bands.

PNG 003.7+00.5 (Figure 3) is another small extended 8.3/21.3- $\mu \mathrm{m}$ source, this time associated with an $\mathrm{H} \alpha$ image only $10^{\prime \prime}$ across, and showing $\mathrm{H} \alpha$, [NII], and [SII]. Note the close coincidence of 8.3 and $21.3-\mu \mathrm{m}$ emission.

Wolf-Rayet central stars of PN are rather rarely encountered (only 56 were known in the galaxy). However, Morgan \& Parker (these proceedings) have reported a total of 7 new such objects discovered from spectroscopic follow-up of the new $\mathrm{H} \alpha$ survey candidates. Figure 4 illustrates one of these, PN PMR4, with its WC8 CSPN. The MIR image shows more extensive, bright $21.3-\mu \mathrm{m}$ emission compared with the $8.3 \mu \mathrm{m}$ image.

In Figure 5 we present G321.3-00.3, still a PN candidate because, as yet, we lack definitive optical spectroscopy to assign the object to PN status. This 


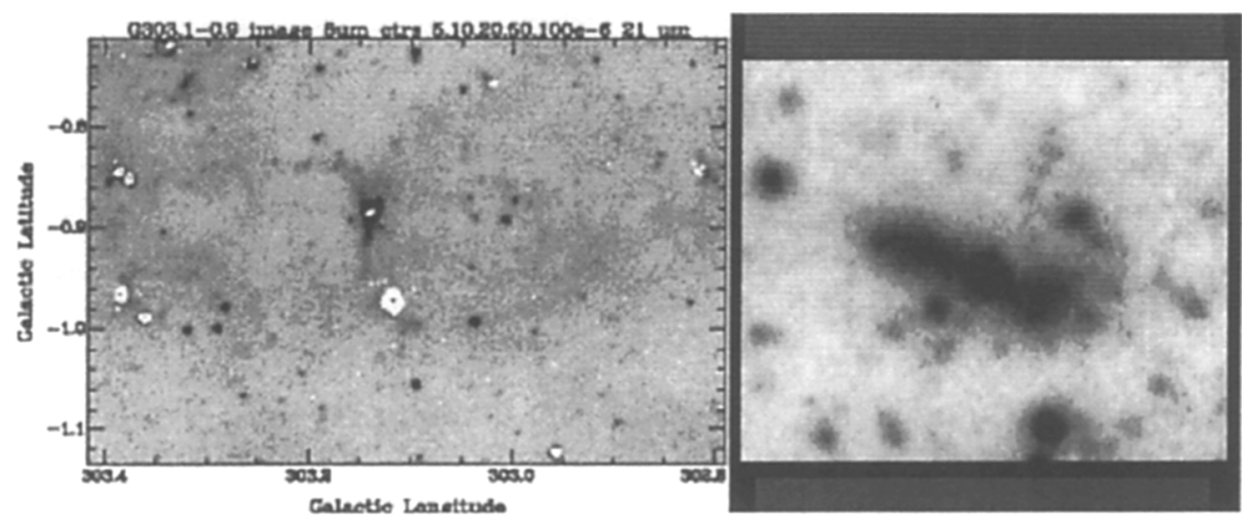

Figure 2. The bipolar object, PNG 303.1-00.9, in greyscale at $8.3-\mu \mathrm{m}$ with white contours of $21.3-\mu \mathrm{m}$ emission (left), and $\mathrm{H} \alpha$ (right).

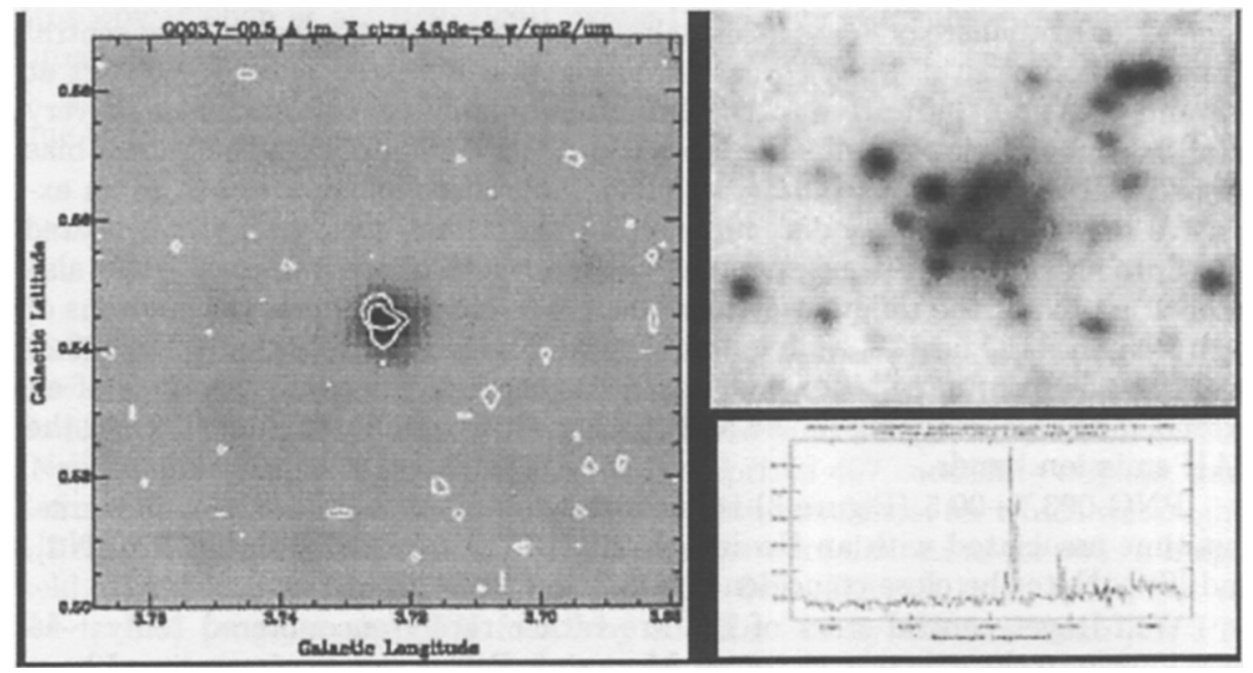

Figure 3. PNG 003.7+00.5: 8.3- $\mu \mathrm{m}$ and $\mathrm{H} \alpha$ images, and the red spectrum. 


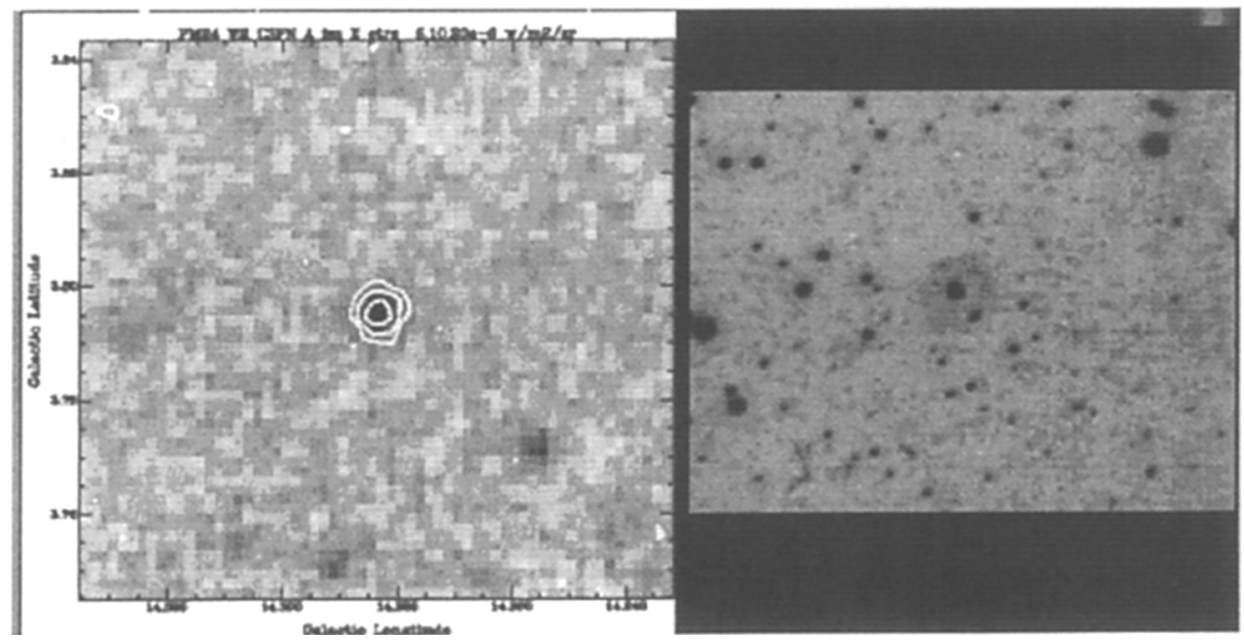

Figure 4. The PN PMR4 and its WC8 nucleus at 8.3/21.3 $\mu \mathrm{m}$ (left) and in $\mathrm{H} \alpha$ (right).

is a sizeable (94" diameter), circular nebula showing only $\mathrm{H} \alpha$ and [NII emission lines. By contrast, the $8.3-\mu \mathrm{m}$ map shows a distinct central hole, $\sim 90^{\prime \prime}$ across, suggestive of a MIR PDR surrounding the inner, ionized gas region. $21.3-\mu \mathrm{m}$ emission is also seen in this central hole, indicative of dust mixed with the ionized gas (the complex appearance is due to the projection of at least two compact HII regions against the main nebula).

\section{MSX \& star-forming regions}

During our study of $\mathrm{H} \alpha$ survey PN candidates, we often encounter regions of the plane in which MSX shows abundant MIR objects, some point sources, others consisting of faint diffuse 8.3- $\mu \mathrm{m}$ emission. One has the distinct impression that these nebulosities are indications of embedded star-forming regions, likely of intermediate mass stars, perhaps in local clusterings. In such environments we are less likely to characterize $\mathrm{H} \alpha$ nebulae as being potential PN. An example of such a region is illustrated by Figure 6. Our particular sub-sample of random $\mathrm{H} \alpha$ candidates close to the plane appears to consist entirely of reflection nebulae, compact HII regions, and embedded objects.

\section{Eliminating (ultra)compact HII regions using MSX}

G301.5+00.7 (Figure 7) was an early PN candidate but the great MIR brightness, unusual morphology, extended low-surface-brightness diffuse emission at $8.3 \mu \mathrm{m}$, yet relatively compact $21.3 \mu \mathrm{m}$ emission core all mitigate against a PN classification. Our optical spectra show a strong continuum, also unlike the weak 2-photon and/or bound-free emission shown by the spectra of true PN. 


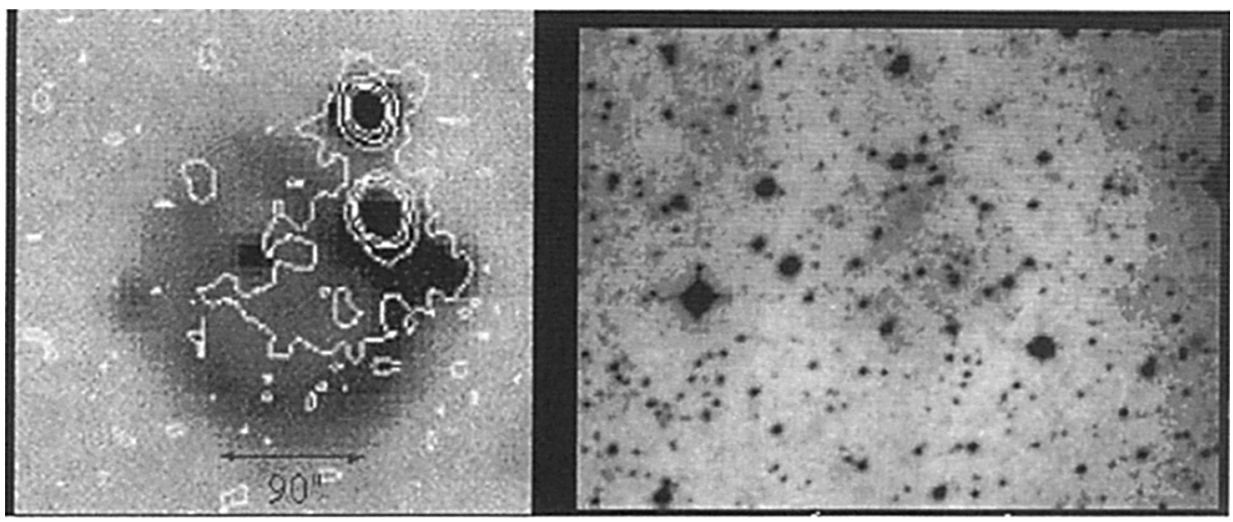

Figure 5. The sizeable ring nebula, G321.3-00.3, with the 8.3- $\mu \mathrm{m}$ central hole that matches the ionized gas shown in the $\mathrm{H} \alpha$ image.
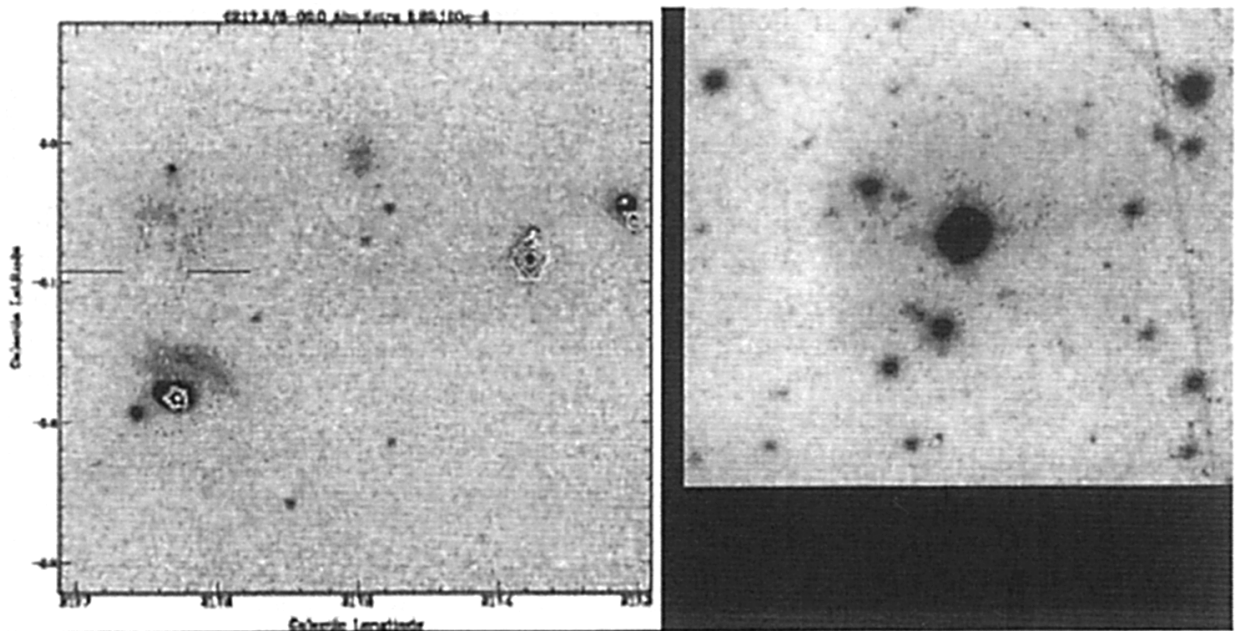

Figure 6. A region of the plane containing several MIR nebulae, most likely due to embedded young stars. The PN candidate's location is between the two bars in the left image but no MIR counterpart is seen. 


\section{The statistics of MSX counterparts to true \& probable PN}

Overall, $34 \%$ of the $142 \mathrm{PN}$ candidates that we have surveyed with MSX have MIR counterparts. However, restricting the sample solely to true and probable PN we find $18 \%$ of 74 MSX detections, with the following breakdown: only 2 of 28 candidate $\mathrm{PN}$ in the bulge field; 3 of 19 in the $l=300^{\circ}$ field; none of the large PN; 2 out of the 10 bona-fides; 2 of 3 PN with Wolf-Rayet central stars observed by MSX; 4 of $10 \mathrm{PN}$ with interesting $\mathrm{H} \alpha$ morphologies, all bipolar, showing the bias toward bipolarity among MIR objects detected; and none of the $b \sim 0^{\circ}$ subset. Small optical nebulae are also more likely to be detected by MSX (unless in the Bulge).

\section{Why does MSX not detect more true $\mathrm{PN}$ from the $\mathrm{H} \alpha$ survey?}

If we directly compare the Acker et al (1992) catalogue of $1100 \mathrm{PN}$ with the MSX Point Source Catalog (Egan et al. 1999), which is derived from the Galactic Plane imagery, we find that of order $35 \%$ of Acker PN are matched with MSX objects, a substantial "hit rate" given the $|b| \leq 5^{\circ}$ limit of MSX ( $>90 \%$ of known Galactic PN have $|b| \leq 20^{\circ}$ ). Hence why is the hit rate so low with the new $\mathrm{H} \alpha$ survey's true and probable PN? We offer the following possible explanations.

In general, $\mathrm{PN}$ that are too distant or are confused by high point source density (e.g., in the bulge), are less likely to be detected by MSX's survey of the plane. There are several hundred new PN in this category alone. Because the primary emission mechanism in the MIR is thermal emission by dust, any PN with too little, or no, dust, or with a dust temperature that is too low to be readily detected at SPIRIT-III's longest wavelength $(21.3 \mu \mathrm{m})$ would not have been well detected by MSX. If the nebular electron density is too low to trap Ly $\alpha$ photons, then dust grains absorb only dilute starlight, compromising the detectability of low-density nebulae with MSX. Similarly, any evolved nebula with a low-luminosity star is hard to detect in the MIR. Again the new $\mathrm{H} \alpha$ survey is revealing a substantial population of evolved PN. If a PN has too little UV radiation to pump $\mathrm{PAH}$ molecules, or these molecules are destroyed at the interface with the ISM or by external events, such PN also would elude detection by the MSX survey.

\section{Conclusions}

When applied to the specific PN candidates discovered by the AAO/UKST H $\alpha$ survey, we feel that the MSX imaging survey of the plane can play a significant role in: (i) recognizing non-PN by their MIR morphology and ratios of MIR/radio spatially-integrated source density (see Cohen \& Green 2001) especially when optical morphology and spectroscopy are inconclusive; (ii) suggesting the presence of edge-on disks (typically inferred for nebulae with a bipolar morphology) by their MIR spectral energy distributions (i.e. by the implied wide range in dust temperature); (iii) understanding the physical processes at interfaces with the ISM (e.g. PDRs); (iv) identifying PAH or fine-structure line emission from comparison of images in $4 \mathrm{MSX}$ bands: i.e., identical morphologies at 8.3 and $12.1-\mu \mathrm{m}$ suggest $\mathrm{PAH}$ emission, while very different morphologies 

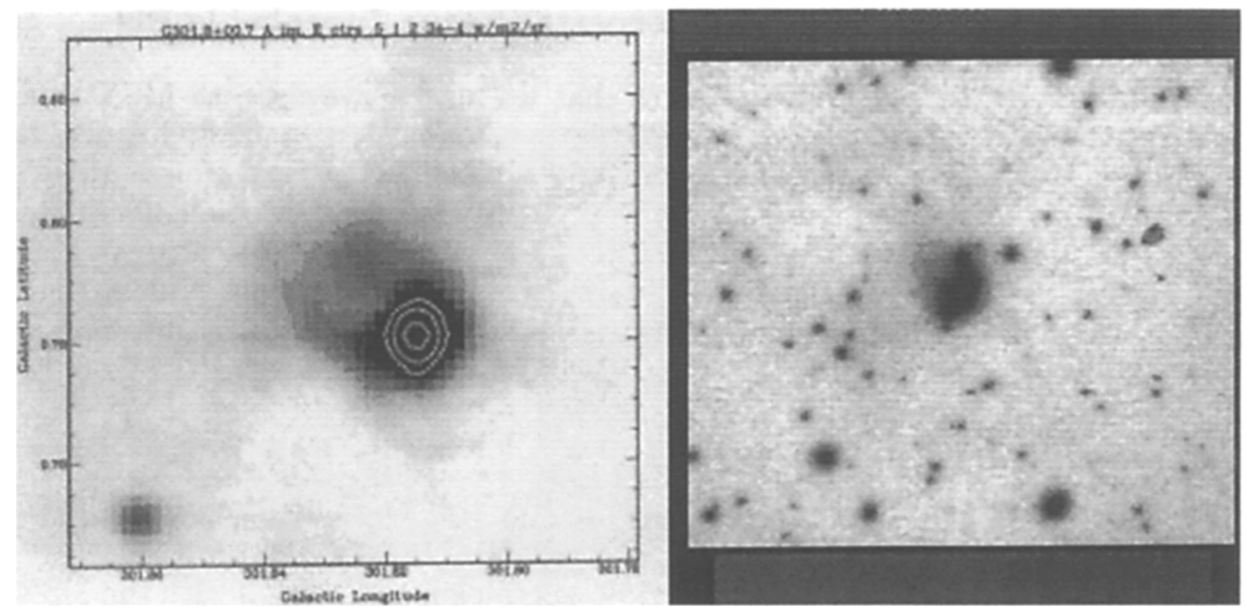

Figure 7. The MIR morphology and optical spectra suggest G301.5+00.7 is a compact HII region not a PN.

might indicate emission lines; similar morphologies at 12.1 and $21.3 \mu \mathrm{m}$ make thermal emission by dust the likeliest mechanism.

Acknowledgments. MC acknowledges NASA support under LTSA grant NAG5-7936 with Berkeley.

\section{References}

Aitken, D.K., Roche, P.F., Spenser, P.M., Jones, B. 1979, ApJ, 233, 925

Casassus, S., Roche, P.F., Aitken, D.K., Smith, C.H. 2001, MNRAS, 327, 744

Cohen, M., Allamandola, L.J., Tielens, A.G.G.M., Bregman, J., Simpson, J.P., Witteborn, F.C., Wooden, D., Rank, D. 1986, ApJ, 302, 737

Cohen, M. \& Barlow, M.J. 1974, ApJ, 193, 401

Cohen, M. \& Barlow, M.J. 1980, ApJ, 238, 585

Cohen, M. \& Green, A. 2001, MNRAS, 325, 531

Cohen, M., Tielens, A.G.G.M., Bregman, J., Witteborn, F.C., Rank, D., Allamandola, L.J., Wooden, D., de Muizon, M. 1989, ApJ, 341, 246

Cox, P. et al. 1998, ApJ, 495, 23

Egan, M.P. et al. 1999, "The Midcourse Space Experiment Point Source Catalog Version 1.2 Explanatory Guide", Air Force Research Laboratory Technical Report, AFRL-VS-TR 1999-1522

Mill, J., O’Neil, R.R., Price, S.D., Romick, G.J., Uy, O.M., Gaposchkin, E.M. 1994, AIAA, 31, 900

Price, S.D., Egan, M.P., Carey, S., Mizuno, D., Kuchar, T. 2001, AJ, 121, 2819

Volk, K. \& Cohen, M. 1990, AJ, 100, 485 